\title{
A NON-SUBSTRATE EFFECTOR OF P-HYDROXYBENZOATE HYDROXYLASE
}

\author{
Larry G. Howell and Vincent Massey \\ Department of Biological Chemistry \\ The University of Michigan
}

Ann Arbor, Michigan 48104

Received July 7, 1970

SUMMARY: A substrate analogue, 6-hydroxynicotinate (6-OHNA)*, facilitates reduction of the enzyme bound flavin by TPNH but is not hydroxylated in the reoxidation of the enzyme by oxygen. Binding of 6-OHNA to oxidized p-hydroxybenzoate hydroxylase is accompanied by a perturbation of the visible spectrum and enhancement of the flavin fluorescence. Titrations using these properties indicate the formation of a 1:1 complex with a dissociation constant of $2.5 \times 10^{-4} \mathrm{M}$. At high concentrations of 6 -OHNA, binding of 6-OHNA to additional site (s) has been observed. The 6-OHNA binding is competitive with p-hydroxybenzoate binding as indicated by the reversal of p-hydroxybenzoate quenching of flavin fluorescence on addition of 6-OHNA. The presence of the effector, 6-OHNA, is necessary for the catalytic oxidation of TPNH.

Previous work has demonstrated the role of p-hydroxybenzoate as both a substrate and an effector of p-hydroxybenzoate hydroxylase. In addition to the dependence of TPNH oxidation on the concentration of p-hydroxybenzoate $1,2,3$ Hosokawa and stanier demonstrated that p-hydroxybenzoate hydroxylase catalyzed the anaerobic rapid reduction of exogenous FAD only in the presence of p-hydroxy. benzoate $^{1}$. Similar properties have been observed, both aerobically and anaerobically, with the p-hydroxybenzoate hydroxylase catalyzed reduction of ferricyanide by TPNH ${ }^{4}$, which also requires the presence of p-hydroxybenzoate for rapid reduction of fexricyanide. Stopped flow studies have shown that the rate of anaerobic reduction of the enzyme bound flavin is greatly enhanced by the presence of p-hydroxybenzoate $2,3,5,6$. The binding of p-hydroxybenzoate to p-hydroxybenzoate hydroxylase has been found to bring about conformational changes in the protein structure as indicated by changes in crystal form ${ }^{7}, \mathrm{ORD}^{\circ}$ changes $^{7}, C D$ changes ${ }^{8}$ and a change in the sedimentation coefficient upon binding of p-hydroxybenzoate ${ }^{7}$. Although some aromatic compounds have been shown to inhibit the catalytic oxidation of $\mathrm{TPNH}^{1,8}$, none of the above mentioned properties associated with the effector role of p-hydroxybenzoate have been observed with p-hydroxybenzoate analogues. This paper presents evidence that 6-OHNA, not itself a substrate, acts as an effector upon p-hydroxybenzoate hydroxylase

*The following abbreviations are used: reduced triphosphopyridine nucleotide;
6-OHNA, 6-hydroxynicotinate; TPNH, $F A D$, flavin adenine dinucleotide. 
isolated from Pseudomonas fluorescens ${ }^{2}$.

RESULTS

Catalytic properties: 6-hydroxynicotinate, a close structural analogue of p-hydroxybenzoate, was found to induce TPNH oxidoreductase activity with p-hydroxybenzoate hydroxylase, employing $\mathrm{O}_{2}$ or $\mathrm{K}_{3} \mathrm{Fe}(\mathrm{CN})_{6}$ as electron acceptor. Low concentrations of 6-OHNA were found to permit the oxidation of much higher concentrations of TPNH, suggesting that it was an activator (ie. an effector) of the enzyme rather than a true substrate. This role as an effector was confirmed by the steady state analysis of the TPNH-oxygen reductase activity (Fig. 1) which satisfies an equation of the form:

$$
\text { eq. } 1 \quad \frac{1}{V}=\frac{1}{V}\left(1+\frac{K_{S}}{S}\right)+\frac{K_{A}}{A \cdot V} \cdot \frac{\left(1+K_{S} / S\right)}{\left(1+S / K_{S}\right)}
$$

where $S$ stands for the concentration of TPNH, A for the concentration of 6-OHNA, $\mathrm{K}_{\mathrm{S}}$ for the Michaelis constant for $\mathrm{TPNH}$ and $\mathrm{K}_{\mathrm{A}}$ for the dissociation constant of the enzyme-6-OHNA complex.
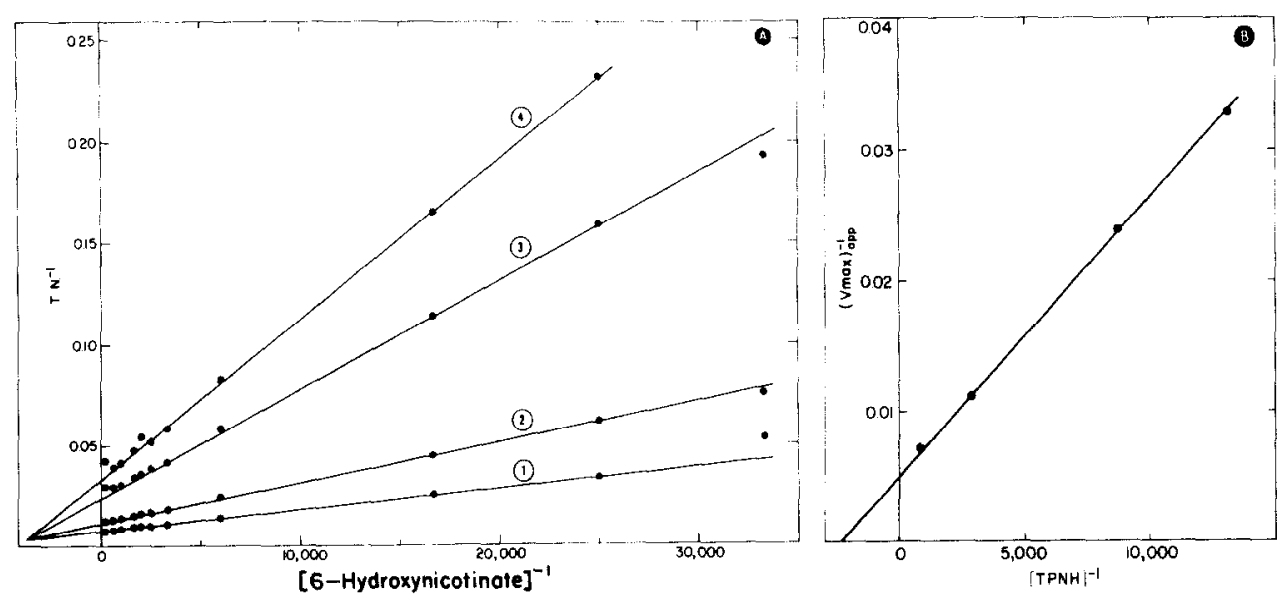

Fig. 1. Effect of 6-OHNA on TPNH oxidase activity of p-hydroxybenzoate hydroxylase. A. At concentrations of TPNH of $1.14 \times 10^{-3} \mathrm{M}, 3.42 \times 10^{-4} \mathrm{M}$, $1.14 \times 10^{-4} \mathrm{M}$ and $7.6 \times 10^{-5} \mathrm{M}$ for lines $1-4$. B. Secondary plot of $\mathrm{y}$-intercepts from $\mathrm{A}$. Conditions: $0.02 \mathrm{M}$ Tris: $\mathrm{KH}_{2} \mathrm{PO}_{4} \mathrm{pH} 7.6,3 \times 10^{-4} \mathrm{M}$ EDTA, $25^{\circ}$. Velocities expressed as turnover number, ie., moles TPNH oxidized per minute per mole enzyme bound flavin. 
This equation is consistent with a catalytic mechanism in which substrate is bound only to the enzyme-activator complex and the rate of reduction of the enzyme by the substrate is the rate limiting step ${ }^{9}$. In this case, the $x$-coordinate of the common point of intersection (Fig. 1) is equal to the negative reciprocal of the dissociation constant for the activator. The value for the dissociation constant, $\mathrm{K}_{\mathrm{A}}$, as calculated from the data of Fig. $1 \mathrm{~A}$, is $2.66 \times 10^{-4} \mathrm{M}$. From the secondary plot of the $\mathrm{Y}$-intercepts of $\mathrm{Fig} .1 \mathrm{~A}, \mathrm{~K}_{\mathrm{s}}$ and $v_{\max }$ may be determined. The $x$-intercept of the secondary plot (Fig. 1B) is equal to the negative reciprocal of $\mathrm{K}_{\mathrm{s}}$; the $\mathrm{y}$-intercept is equal to the reciprocal of the maximum velocity. From the data of Fig. $1 \mathrm{~B}, \mathrm{~K}_{\mathrm{S}}=4.35 \times 10^{-4} \mathrm{M}$ and $V_{\max }=202$ moles of TPNH oxidized per min per mole of enzyme.

It should be noted that the common point of intersection in Fig. IA is above the $x$-axis, not on the $x$-axis as would have been the case if the binding of TPNH was independent of 6-OHNA (Eq. 2).

$$
\text { eq. } 2 \quad \frac{1}{V}=\frac{1}{V}\left(1+\frac{K_{S}}{S}\right)+\frac{K_{A}}{A \cdot V}\left(1+\frac{K_{S}}{S}\right)
$$

It is also apparent from Fig. IA that high concentrations of 6-OHNA are inhibitory.

Fate of 6-OHNA: Having observed that 6-OHNA was necessary for the p-hydroxybenzoate hydroxylase catalyzed oxidation of TPNH by $\mathrm{O}_{2}$, it remained necessary to determine the extent of modification of the 6-OHNA during catalytic turnover. This was investigated using gel filtration to separate the reaction products. The reaction mixture contained TPNH, $10^{-3} \mathrm{M} ; 6$-OHNA, $4 \times 10^{-4} \mathrm{M}$; FAD, $3.33 \times 10^{-6} \mathrm{M}$; buffer, $0.02 \mathrm{M}$ tris: $\mathrm{KH}_{2} \mathrm{PO}_{4}$ plus $3 \times 10^{-4} \mathrm{M}$ EDTA, pH 7.6 . A zero time aliquot was withdrawn before addition of the enzyme. Enzyme was then added ( 0.86 units per $\mathrm{ml})$ and the reaction followed to completion by following the decrease in absorbance at $373 \mathrm{m \mu}$. Aliquots $(0.5 \mathrm{ml})$ were applied to a Bio-Gel P-2 column, I x $47 \mathrm{~cm}$, equilibrated with $0.02 \mathrm{M}$ tris: $\mathrm{KH}_{2} \mathrm{PO}_{4}$ plus $3 \times 10^{-4} \mathrm{M}$ EDTA, $\mathrm{pH} 7.6$, and eluted with the starting buffer. The 6-OHNA peak isolated from the final reaction mixture possessed the same spectral characteristics $\left(A_{250}: A_{294}=2.31\right)$ as the zero time sample $\left(A_{250}: A_{294}=2.36\right.$; theoretical $=2.34)$. The recovery of 6 -OHNA was 988 (calculated on basis of $\varepsilon_{294}=$ $5.4 \times 10^{3} \mathrm{M}^{-1} \mathrm{~cm}^{-1}$ at $\mathrm{pH} 7.6$ ). This is consistent with the initial observation that limited amounts of 6-OHNA facilitate the oxidation of much larger amounts of TPNH.

Binding of 6-OHNA by $p$-hydroxybenzoate hydroxylase: Addition of 6-OHNA to p-hydroxybenzoate hydroxylase is accompanied by spectral shifts similar to those observed on binding of p-hydroxybenzoate $1,2,5,7,8$. The enzyme was titrated with 6-OHNA using Yankeelov cuvettes ${ }^{10}$, obtained from the Pyrocell Manufacturing Company, Westwood, New Jersey. The difference spectra obtained 

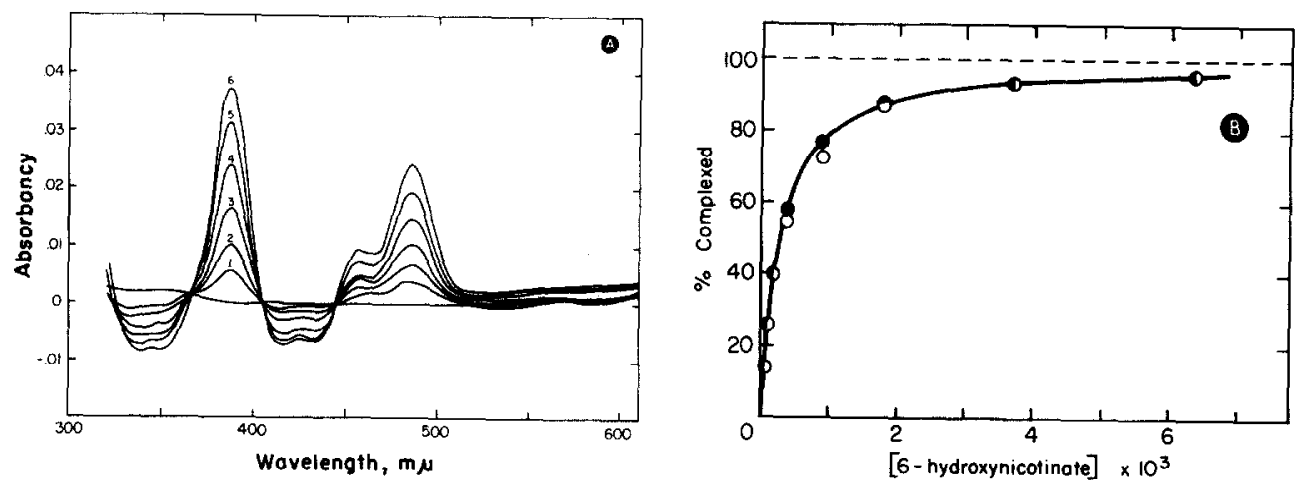

Fig. 2. Spectrophotometric titration of p-hydroxybenzoate hydroxylase with 6-OHNA. A. Difference spectra of p-hydroxybenzoate hydroxylase plus 6-OHNA versus enzyme without effector. Conditions as in Fig. 1, enzyme concentration, $5.80 \times 10^{-5} \mathrm{M}$. Curves $1-6$ contained respectively $4.99 \times 10^{-5} \mathrm{M}, 9.96 \times 10^{-5} \mathrm{M}$, $1.99 \times 10^{-4} \mathrm{M}, 3.97 \times 10^{-4} \mathrm{M}, 7.90 \times 10^{-4} \mathrm{M}, 3.65 \times 10^{-3} \mathrm{M} 6$-OHNA. Sample compartment has a light path of $4.5 \mathrm{~mm}$. B. - theoretical curve calculated for a dissociation constant of $2.51 \times 10^{-4} \mathrm{M} ; \bullet$, experimental data using the change in absorbance at $387 \mathrm{~m} \mu$; 0 , experimental data using the change in absorbance at $485 \mathrm{mu}$.

are shown in Fig. 2A. These data revealed that a $1: 1$ complex was formed, with a dissociation constant of $2.51 \times 10^{-4} \mathrm{M}$. The fit of the experimental points to the theoretical curve is shown in Fig. $2 \mathrm{~B}$.

Addition of 6-OHNA to p-hydroxybenzoate hydroxylase is also accompanied by a 1.8 fold enhancement of the enzyme bound flavin fluorescence to a level which is 228 greater than the fluorescence of free FAD under the same conditions. At concentrations of 6-OHNA below $10^{-3} \mathrm{M}$, the enhancement of the enzyme flavin fluorescence by 6-OHNA followed a $1: 1$ binding pattern, with a dissociation constant of $2.56 \times 10^{-4} \mathrm{M}$, as determined from a Benesi-Hildebrand plot ${ }^{11}$ (Fig. 3, inset). At higher concentrations of 6-OHNA, deviation from the theoretical 1:1 binding was observed. This deviation at high concentrations of 6-OHNA is accompanied by excess substrate inhibition in catalytic turnover experiments, as has also been observed with p-hydroxybenzoate ${ }^{1,4}$, and is due to the binding of a second molecule of 6-OHNA.

It has also been found that binding of p-hydroxybenzoate results in a 75\% decrease in the flavin fluorescence of p-hydroxybenzoate hydroxylase 4 . 


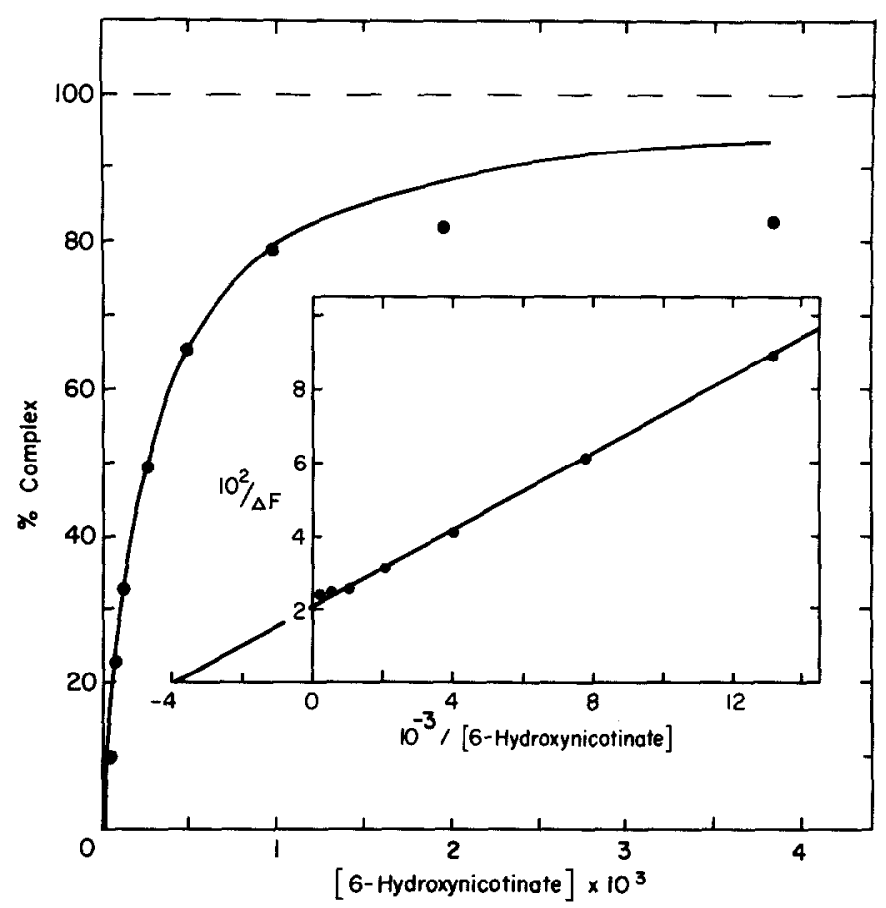

Fig. 3. Fluorometric titration of p-hydroxybenzoate hydroxylase with 6-OHNA. Conditions as in Fig. 1; enzyme concentration, $5.42 \times 10^{-6} \mathrm{M}$. , theoretical curve calculated for a dissociation constant of $2.56 \times 10^{-4} \mathrm{M}$; - experimental data obtained from fluorescence measurements. Inset, BenesiHildebrand plot ${ }^{11} ; \Delta F$, change in fluorescence using arbitrary fluorescence units. Excitation at $450 \mathrm{m \mu}$; emission at $525 \mathrm{m \mu}$.

Preliminary fluorescence studies indicate that the binding of 6-OHNA is competitive with that of p-hydroxybenzoate.

Direct studies on the rate of TRNH reduction of the enzyme bound flavin: The kinetic and binding studies presented in previous sections indicate very strongly the effector role of p-hydroxybenzoate and 6-OHNA. Direct evidence for this role as an effector was obtained by rapid reaction spectrophotometry, where the rate of reduction of the enzyme-bound flavin was followed. The rate at which the enzyme-6-OHNA complex is reduced by TPNH is markedly increased relative to the rate of reduction of the holoenzyme in the absence of effector. The p-hydroxybenzoate hydroxylase-p-hydroxybenzoate complex is reduced by TPNH at an even greater rate with an apparent $k_{\text {red }}$ approximately 980 times greater than the apparent $k_{\text {red }}$ of the 6-OHNA complex. These data are summarized in Table 1 .

In addition to the difference in magnitude of the rate constants as indicated in Table 1 , it is important to note the relative values of $k_{r e d}$ and the 
Table 1

Stimulation of Anaerobic Reduction of Enzyme-Bound Flavin by Effectors

\begin{tabular}{lcc} 
Conditions & $\begin{array}{c}\text { Rate of reduction } \\
\text { of enzyme flavin* }\end{array}$ & $\begin{array}{c}\text { Observed catalytic } \\
\text { velocity }\end{array}$ \\
\hline TPNH, $10^{-3} \mathrm{M}$ & $0.1045 \mathrm{~min}^{-1}$ & none \\
\hline $\begin{array}{l}\text { TPNH, } 10^{-3} \mathrm{M} \\
6-\mathrm{OHNA}, 2 \times 10^{-3} \mathrm{M}\end{array}$ & $122 \mathrm{~min}^{-1}$ & $118 \mathrm{~min}^{-1}$ \\
\hline TPNH, $5 \times 10^{-4} \mathrm{M}$ & $12,480 \mathrm{~min}^{-1}$ & $1,371 \mathrm{~min}^{-1}$ \\
p-hydroxybenzoate, \\
$2.5 \times 10^{-4} \mathrm{M}$
\end{tabular}

*Determined at p-hydroxybenzoate hydroxylase concentrations between $1.09 \times 10^{-5} \mathrm{M}$ and $1.66 \times 10^{-5} \mathrm{M}, \mathrm{pH} 7.6,25^{\circ}$, by following the decrease in absorbance at $460 \mathrm{m \mu}$, employing the stopped-flow spectrophotometer of Gibson and Milnes. 12

observed catalytic velocity. While previous work with this enzyme has demonstrated that $\mathrm{k}_{\text {red }}$ is not the rate limiting step when $\mathrm{p}$-hydroxybenzoate is the effector, $k_{r e d}$ does seem to be the rate limiting step when 6-OHNA is the effector. This is consistent with the steady state analysis of the 6-OHNA system presented above.

\section{DISCUSSION}

The above results document the role of 6-OHNA as a non-substrate effector of p-hydroxybenzoate hydroxylase. A similar uncoupling phenomenon has recently been observed with salicylate hydroxylase ${ }^{13}$. The $v_{\max }$ of the benzoate-stimulated oxidation of DPNH by salicylate hydroxylase is the same as the $v_{\max }$ for salicylate hydroxylation, while the $v_{\max }$ with 6-OHNA is less than $10 \%$ of the $\mathrm{V}_{\max }$ for $\mathrm{p}$-hydroxybenzoate hydroxylation. The steady state analysis presented above also indicates that the dissociation constant of TPNH and p-hydroxybenzoate hydroxylase is significantly affected by the effector molecule. This conclusion is similar to that of White-Stevens and Kamin ${ }^{13}$ that both salicylate and benzoate facilitate DPNH binding to salicylate hydroxylase. The data presented above strongly indicate that, with p-hydroxybenzoate hydroxylase from Pseudomonas fluorescens, the role of the effector is to facilitate both the reduction of the enzyme flavin by TPNH and the binding of TPNH. Rapid reaction studies are in progress to determine if the observed effect of 6-OHNA on the Michaelis constant for TPNH is indeed due to a change in the dissociation constant of the enzyme TPNH complex. 
Supported by grants GM 25736 and GM 11106 from the U.S. Public Health Service.

\section{REFERENCES}

1. Hosokawa, K. and Stanier, R. Y., J. Biol. Chem., 241, 2453 (1966).

2. Howell, I. G. and Massey, V., in H. Kamin (Editor) "Flavins and Flavoproteins, Proceedings of the Third International Symposium", University Park Press, Baltimore, 1970 (in press).

3. Yano, K., Higashi, N., Nakamura, S. and Arima, K., Biochem. Biophys. Res. Comm., 34, 277 (1969).

4. Howell, L. G., unpublished observations.

5. Massey, V., Matthews, R. G., Foust, G. P., Howell, I. G., Williams, C. H., Jr., Zanetti, G., and Ronchi, S., in H. Sund (Editor) "Pyridine NucleotideDependent Dehydrogenases, Proceedings of an Advanced Study Institute, Konstanz, Germany, September, 1969", Springer-Verlag, Berlin, 1970, p. 393.

6. Yano, K., Higashi, N., Nakamura, S. and Arima, K., Agr. Biol. Chem., 33, 1363 (1969).

7. Yano, K., Higashi, N. and Arima, K., Biochem. Biophys. Res. Comm., 34, 1 (1969).

8. Hesp, B., Calvin, M. and Hosokawa, K., J. Biol. Chem., 244, 5644 (1969).

9. Dixon, M., and Webb, E. C., "Enzymes", Academic Press, Inc. (New York) (1964) p. 430.

10. Yankeelov, J. A., Jr., Anal. Biochem., 6, 287 (1963).

11. Benesi, H. A. and Hildebrand, T. A., J. Amer. Chem. Soc., 71, 2703 (1949).

12. Gibson, Q. H. and Milnes, L., Biochem., 91, 161 (1964).

13. White-Stevens, R. H. and Kamin, H., Biochem. Biophys. Res. Comm., 38, $882(1970)$. 